\title{
Femoral Nerve Palsy Following Delayed Reduction of a Dislocated Hip in a 44-Year-old Man
}

\author{
Hassan Rahimi Shorin ${ }^{1}$; Mohammad Azizbeig Mohajer ${ }^{2}$; Ali Parsa ${ }^{3,{ }^{*}}$; Amin Azhari ${ }^{4}$; \\ Maryam Assadian ${ }^{1}$ \\ ${ }^{1}$ Department of Orthopaedic Surgery, Mashhad University of Medical Sciences, Mashhad, IR Iran \\ ${ }_{2}^{2}$ Department of Orthopaedic Surgery, Graz University, Graz, Austria \\ ${ }^{3}$ Department of Orthopaedic Surgery, Zahedan University of Medical Sciences, Zahedan, IR Iran \\ ${ }^{4}$ Department of Medical physics and Rehabilitation, Mashhad University of Medical Sciences, Mashhad, IR Iran \\ *Corresponding Author: Ali Parsa, Department of Medical Physics and Rehabilitation, Mashhad University of Medical Sciences, Mashhad, IR Iran. Tel: +98-9155056358, E-mail: ali- \\ parsadr@yahoo.com
}

Received: May 29, 2013; Revised: August 2, 2013; Accepted: August 21, 2013

Introduction: Incidence of nerve injury in traumatic hip dislocations is up to $10 \%$. Sciatic nerve is the most common injured nerve in this setting. In the medical literature, there are few documented cases of femoral nerve injury following hip dislocations.

Case Report: We report a 44-year-old man with right femoral nerve palsy following delayed reduction of an anterior dislocation of hip. Conclusion: Two months after closed reduction, complete clinical recovery of right femoral nerve was achieved and the patient was able to resume his job.

Keywords:Dislocation; Hip; Femoral Neuropathy

\section{Introduction}

Hip dislocation is either congenital or traumatic. Congenital dislocation of the hip is due to acetabular dysplasia as a result of immature acetabulum in infants, proximal femoral deformities, or dysplasia of the femoral head or acetabulum. On the other hand, traumatic dislocation often occurs during high-speed or high-energy accidents such as road-traffic accidents. A traumatic hip dislocation alone or with accompanying hip fracture is an orthopedic emergency, and it is advocated that an early reduction might reduce complications $(1,2)$. According to the previous studies, incidence of nerve injury in traumatic hip dislocations is up to $10 \%$ and sciatic nerve is the most common injured nerve. In the medical literature, there are few documented cases of femoral nerve injury following hip dislocations. We report a case of femoral nerve palsy secondary to hip dislocation.

\section{Case Report}

In April 2012 a 44-year-old man pedestrian was referred to our hospital (Khatam-al-anbia, Zahedan, Iran) 18 hours after a motor-vehicle accident. First, He was transferred to the nearest hospital, but his dislocation was missed on initial X-ray images (Figure 1). Few hours later, a CT scan was performed owing to his increasing pain that revealed an anterior dislocation of his right hip joint (Figure 2). Consequently, he was referred to our center. In Emergency room, his right lower extremity was in external rotation position. He explained an anesthesia in medial side of right leg and ankle. Active extension of the right knee was impossible and the muscle force could not be assessed due to extreme pain. Motor and sensory functions of the sciatic nerve were normal. The right hip was reduced under general anesthesia in the theater and was followed by skin traction. Post-reduction radiographic images demonstrated an appropriate anatomical position of the right reduced hip without any fracture.

After reduction, marked wasting of knee extensor muscles and decreased sensation in the femoral nerve distribution were noted on examination. Electrophysiological studies shown a neurapraxia and the patient was informed that the muscles force would be restored and the numbness would be resolved. On discharge, skin traction for two weeks, gentle range of motion and knee extensors strengthening exercise, and analgesic were prescribed. Three weeks later, there has been a steady improvement in the patient's symptoms. He could extend the right knee against gravity and the sensation was regained in the medial of the right leg. Therefore, gait training was initiated and physical therapy was continued. At two months follow-up, complete clinical recovery

This report and reports like this can improve the practice and help to take better decisions in management of neurological complications in acute dislocations.

Copyright (c) 2014, Iranian Red Crescent Medical Journal; Published by Kowsar Corp. This is an open-access article distributed under the terms of the Creative Commons Attribution License, which permits unrestricted use, distribution, and reproduction in any medium, provided the original work is properly cited. 


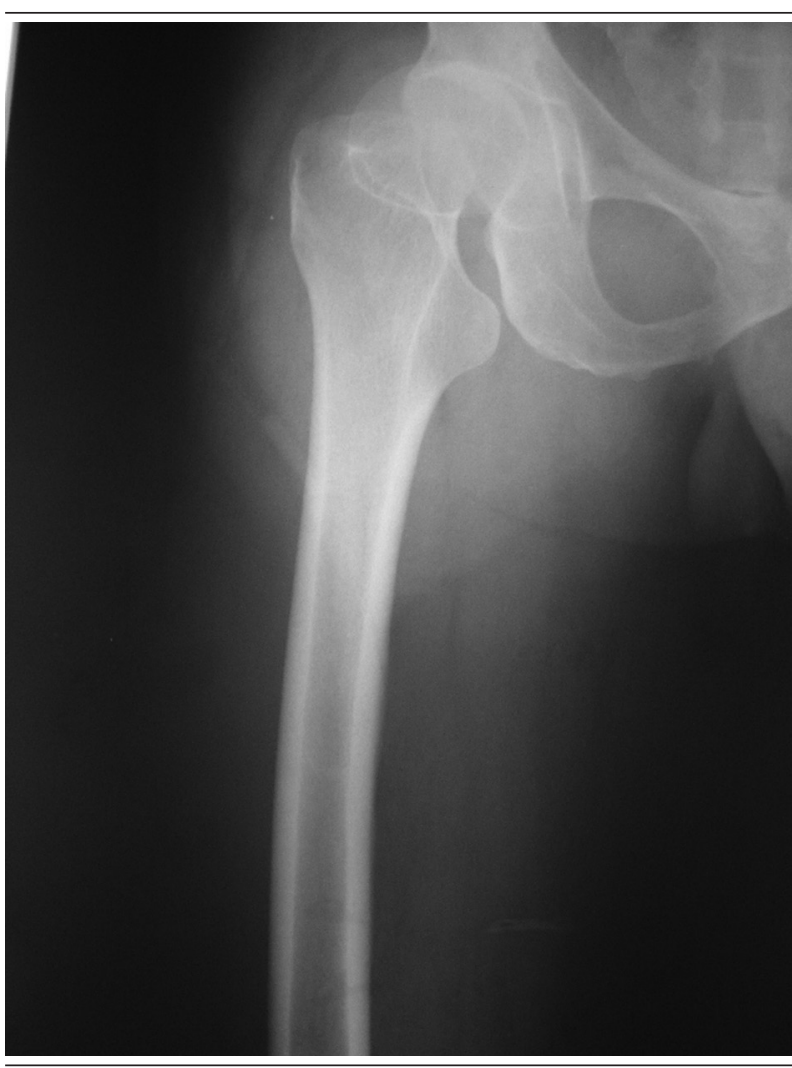

Figure 1. Anterior-Posterior X-ray Images of the Right Hip

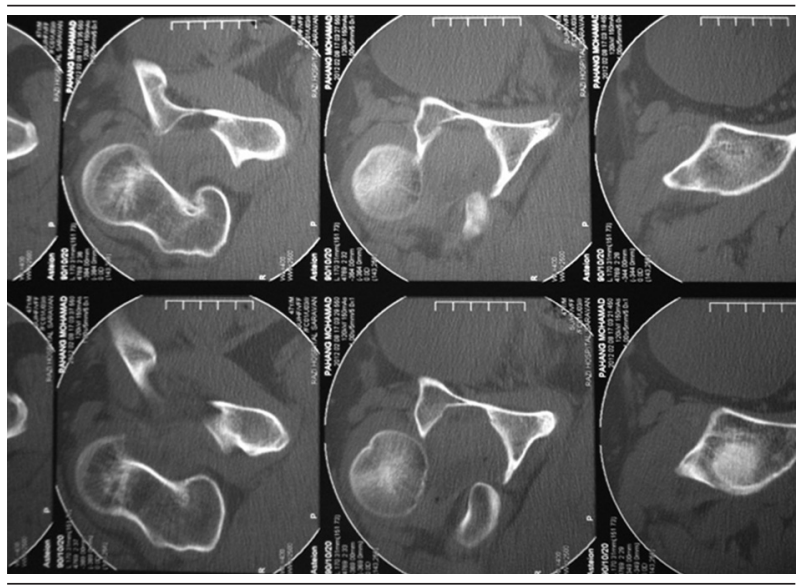

Figure 2. Computed Tomography of the Right Hip

of right femoral nerve was achieved and he was able to resume his job. Findings of an electromyography and nerve conduction study were normal at three months after injury. Recent eight months review has not shown any neurological deficit and he had full range of motion in both right knee and hip joints.

\section{Discussion}

Traumatic dislocation of the hip is an absolute orthopedic emergency. Prompt stable reduction is the key of successful management (3). A delay in detection and reduction leads to irreversible complications (1). While the presentation of sciatic nerve palsy following hip dislocation is well documented, there are few documented cases of femoral nerve palsy following hip dislocation. Other causes of femoral nerve paralysis include pelvic surgeries, retroperitoneal hemorrhage, displaced acetabular fractures (4), iliacus muscle hematoma (5), compartment syndrome (6), open wounds (7), treatment of the hip dysplasia with pavlik harness(8), congenital hip instability (9), and injury during total hip surgery $(10,11)$. The femoral nerve lies medial to the psoas muscle in the same sheath and can be injured with anterior dislocation of hip. In anterior dislocations, the psoas acts as the fulcrum of the hip and the capsule is disrupted both anteriorly and inferiorly. Although rare, in extremely highenergy injuries the femoral neurovascular bundle can be injured or an open dislocation can occur $(9,12)$. Up to December 2012, there were a few published articles concerning femoral nerve palsy secondary to hip luxation. Stein et al. reported hip dislocation of a ballet dancer during a non-contact activity with a post-injury femoral nerve neuropraxia that resolved within six weeks (13). Another patient with a posterior hip dislocation with femoral nerve palsy was reported by Frew et al. (14). In a large series consisted of 726 patients with displaced acetabular fractures, Konrad et al. reported only two cases of posttraumatic femoral nerve palsy (4). However, the average incidence of sciatic nerve injury following hip dislocations is $10 \%$ in the medical literature. Partial recovery of sciatic nerve in these injuries is higher than $60 \%$, although it may not recover completely in delayed reduction (15). It seems that the elapsed time between injury and reduction affects the outcome of nerve palsy. According to this hypothesis a study was conducted by Hillyard et al. that showed transferring of patients with a dislocated hip between hospitals (like our reported patient) have a greater risk of sciatic nerve injury. In this study, the time to reduction was significantly longer in patients with major motor injuries (16). There is discrepancy in the literature regarding to the indications for surgical exploration of nerve injury after hip dislocation; however, most of them recommend that there is no need for surgical treatment unless the patient with normal nerve function experiences deterioration of its function after closed reduction (17). In the presence of nerve palsy, early rehabilitation and follow-up with periodic electromyography studies at one and five months after trauma are recommended (18). It's important to examine neurologic function of the limb before reduction of a dislocation, although examination at the time of injury is difficult. This case emphasizes that early recognition of anterior hip dislocation and prompt closed reduction can relieve distortion of the nerve from a dislocated femoral head and prevent femoral nerve palsy. 


\section{Acknowledgements}

The authors appreciated the help of their colleagues in Mashhad University of Medical Sciences for preparation of this article.

\section{Funding/Support}

There is no funding or supports.

\section{Financial Disclosure}

There is no financial disclosure.

\section{Authors' Contributions}

All the authors did the procedures and follow-up the patient.

\section{References}

1. Sahin V, Karakas ES, Aksu S, Atlihan D, Turk CY, Halici M. Traumatic dislocation and fracture-dislocation of the hip: a long-term follow-up study. J Trauma. 2003;54(3):520-9.

2. Gupta V, Kaur M, Kundu ZS, Kaplia A, Singh D. Traumatic anterior hip dislocation in a 12-year-old child. Chin J Traumatol. 2013;16(2):122-5.

3. Dwyer AJ, John B, Singh SA, Mam MK. Complications after posterior dislocation of the hip. Int Orthop. 2006;30(4):224-7.

4. Gruson KI, Moed BR. Injury of the femoral nerve associated with acetabular fracture. J Bone Joint Surg Am. 2003;85-A(3):428-31.

5. Weiss JM, Tolo V. Femoral nerve palsy following iliacus hematoma. Orthopedics. 2008;31(2):178.
6. Uzel AP, Steinmann G. Thigh compartment syndrome after intramedullary femoral nailing: possible femoral nerve block influence on diagnosis timing. Orthop Traumatol Surg Res. 2009;95(4):309-13.

7. Jobe MT, Martinez SF. Peripheral nerve injuries. In: Canale ST, Beaty J editors. Campbell 's operative orthopaedics. 11th ed: Mosby; 2008. p. 3689.

8. Murnaghan ML, Browne RH, Sucato DJ, Birch J. Femoral nerve palsy in Pavlik harness treatment for developmental dysplasia of the hip. J Bone Joint Surg Am. 2011;93(5):493-9.

9. Firth GB, Mazibuko AD, Munir M. Traumatic dislocation of the hip joint in children. SA Orthopaedic J. 2010;9(1):68-71.

10. Holzapfel BM, Heinen F, Holzapfel DE, Reiners K, Noth U, Rudert M. [Nerve lesions after minimally invasive total hip arthroplasty]. Orthopade. 2012;41(5):354-64.

11. Fritzsche H, Kirschner S, Hartmann A, Hamann C. [Femoral nerve palsy as delayed complication after total hip replacement: delayed hematoma formation in unexpected screw malpositioning]. Orthopade. 2013;42(8):651-3.

12. Schwartz DL, Haller JJ. Open anterior hip dislocation with femoral vessel transection in a child. J Trauma. 1974;14(12):1054-9.

13. Stein DA, Polatsch DB, Gidumal R, Rose DJ. Low-energy anterior hip dislocation in a dancer. Am J Orthop (Belle Mead NJ). 2002;31(10):591-4.

14. Frew N, Foster P, Maury A. Femoral nerve palsy following traumatic posterior dislocation of the native hip. Injury. 2013;44(2):261-2.

15. Cornwall R, Radomisli TE. Nerve injury in traumatic dislocation of the hip. Clin Orthop Relat Res. 2000(377):84-91.

16. Hillyard RF, Fox J. Sciatic nerve injuries associated with traumatic posterior hip dislocations. Am J Emerg Med. 2003;21(7):545-8.

17. Dlabach J. Acute dislocations. In: Canale ST, Beaty J editors. Campbell`s operative orthopaedics. 11th ed: Mosby; 2008. p. 3585.

18. Singh AP, Mittal V. Traumatic inferior hip dislocation with ipsilateral open subtrochanteric fracture: a rare case. Injury Extra. 2008;39(12):384-5. 